$04,12,17$

\title{
Анализ структуры композиционных систем с использованием фрактальных характеристик на примере системы $\mathrm{BaTiO}_{3}$-фуллеренол-ЦЭПС
}

\author{
(C) А.Г. Чекуряев ${ }^{1}$, М.М. Сычев ${ }^{1,2}$, С.В. Мякин ${ }^{1}$ \\ ${ }^{1}$ Санкт-Петербургский государственный технологический институт (технический университет), \\ Санкт-Петербург, Россия \\ ${ }^{2}$ Институт химии силикатов им. И.В. Гребенщикова РАН, \\ Санкт-Петербург,Россия \\ E-mail: sergey_mjakin@mail.ru
}

Поступила в Редакцию 12 января 2021 г.

В окончательной редакции 2 февраля 2021 г.

Принята к публикации 4 фревраля 2021 г.

\begin{abstract}
Предложен подход к исследованию взаимосвязи „состав-структура-свойства“ композиционных материалов, основанный на статистическом анализе распределения структурных элементов композита между фрагментами сечения и расчете фрактальных параметров в качестве количественных характеристик структуры материалов. Перспективность данного подхода продемонстрирована на примере анализа микроструктуры композиционных материалов на основе цианэтилового эфира поливинилового спирта (ЦЭПС) с сегнетоэлектрическим наполнителем титанатом бария $\left(\mathrm{BaTiO}_{3}\right)$, модифицируемом осаждением фуллеренола $\mathrm{C}_{60}(\mathrm{OH})_{42}$. Показано, что модифицирование приводит к снижению размаха и стандартного квадратичного отклонения количества частиц между фрагментами композита, увеличению среднего числа частиц во фрагментах, снижению лакунарности заполнения частицами наполнителя полимерной матрицы и росту интенсивности всех максимумов распределения решеточной плотности и корреляционных радиусов начиная со второго максимума. Полученные результаты свидетельствуют о значительном повышении однородности распределения частиц наполнителя в матрице и предотвращении их агломерации, что обеспечивает увеличение диэлектрической проницаемости композитов на порядок и делает перспективным применение предложенного материала в электронных устройствах.
\end{abstract}

Ключевые слова: композиты, однородность, лакунарность, корреляционный радиус, решеточная плотность, фрактальная размерность, диэлектрическая проницаемость.

DOI: 10.21883/FTT.2021.06.50932.002

\section{1. Введение}

Известно, что на наноуровне свойства веществ определяются не только их химическим составом, но и в значительной степени структурой, т.е. взаимным расположением структурных элементов (атомов, функциональных групп) в пространстве, что хорошо описывается параметрами кристаллической решетки. В случае микроструктуры композиционной системы, такое строгое описание невозможно, так как упорядоченность взаимного расположения структурных элементов, в данном случае - фаз, на большом масштабе отсутствует. В то же время, какая-то характеристика микроструктуры необходима, так как свойства материалов во многом зависят от особенностей взаимного расположения их структурных элементов, в частности однородности распределения, взаимного расположения и дистанции между ними. Примерами подобных систем являются частицы наполнителя в полимерной основе, карбидные и/или оксидные фазы в сплавах, частицы керамики в металлокерамике и т. п. В отличие от кристаллической структуры, характеризующейся строго упорядоченной трансляцией элементарной ячейки на весь объем материала, структурная упорядоченность на микроуровне проявляется не столь отчетливо, поскольку такие параметры, как количество ближайших структурных элементов (координационное число) и расстояние между частицами или фрагментами определенной фазы имеют статистический характер. Вместе с тем упорядоченность и закономерность расположения структурных элементов в таких системах могут проявляться на относительно больших масштабах. Этим обусловлена необходимость определения соответствующих количественных характеристик и их взаимосвязи с составом материала, условиями его формирования и целевыми свойствами. Подходами к решению данной задачи являются статистический анализ распределения структурных элементов материала между отдельными фрагментами его объема, а также анализ фрактальных характеристик, характеризующих степень самоподобия (масштабной инвариантности) системы при увеличении масштаба.

В настоящей работе указанный подход был применен по отношению к используемым в современной технике композиционным материалам на полимерной основе с сегнетоэлектрическим наполнителем (гибкой электрони- 
ке, устройствах хранения энергии, дисплеях, конденсаторах и других электронных устройствах), целевой характеристикой которых является высокая диэлектрическая проницаемость.

Свойства материалов данного типа в значительной степени определяются характером распределения частиц наполнителя в полимерной матрице, наличием связей между ними и предотвращением их неконтролируемой агломерации [1]. Для описания характера распределения структурных элементов необходим анализ количественных характеристик взаимного расположения ансамбля частиц наполнителя в полимерной матрице. Для моделирования подобных систем в ряде исследований применяется расчет фрактальных характеристик на основе анализа радиального распределения частиц $[2,3]$. Вместе с тем, важной и во многом нерешенной задачей остается установление взаимосвязи между математическими моделями, описывающими структуру композитов, и их целевыми свойствами. В [4-7] был предложен комплексный подход к управлению электрическими характеристиками полимерно-неорганических композитов, в частности повышению диэлектрической проницаемости и снижению диэлектрических потерь за счет регулирования донорно-акцепторных межфазных взаимодействий в системе наполнитель-связующее, в том числе модифицирования поверхностного слоя сегнетоэлектрических наполнителей наноуглеродными добавками. Максимальные значения диэлектрической проницаемости подобных композитов были достигнуты при модифицировании титаната бария осаждением фуллеренола из водной суспензии. Введение данной добавки в оптимальном количестве обеспечило интенсивное межфазное взаимодействие с полимерной матрицей с участием гидроксильных групп [7]. В другой серии исследований [8-10] структура полимеров и композитов на их основе была изучена методом электронной микроскопии с анализом фрактальных характеристик, что позволило получить представление об особенностях взаимного расположения частиц наполнителя.

В продолжение указанных исследований в данной работе предложен подход к комплексному анализу однородности распределения частиц наполнителя в полимерной матрице во взаимосвязи с целевыми диэлектрическими характеристиками композитов.

\section{2. Экспериментальная часть}

Объектом исследования являлись синтезированные в [7] композиты на основе цианэтилового эфира поливинилового спирта (ЦЭПС) марки PB-КРТ (Shanghai Keyan Phosphor Technology Co, Ltd., Китай) с сегнетоэлектрическим наполнителем титанатом бария (НРВТ-1В) производства Fuji Titanium (Япония) с удельной поверхностью $1.43 \pm 0.07 \mathrm{~m}^{2} / \mathrm{g}$, диаметром частиц 0.5-1 $\mu$ m и диэлектрической проницаемостью $\varepsilon \sim 4400$.
Для сопоставления характеристик распределения наполнителя были выбраны следующие композиты, содержащие данный наполнитель в количестве 20ن்ol.\%:

- с исходным необработанным наполнителем $\left(\varepsilon_{\text {comp. }} \sim 50\right)$;

- с наполнителем, модифицированным осаждением фуллеренола в оптимальном количестве, составляющем 0.32 mass.\% относительно массы $\mathrm{BaTiO}_{3}$. Для данного композита было достигнуто максимальное увеличение диэлектрической проницаемости (до $\varepsilon_{\text {comp. }} \sim 390$ ).

Структуру композитов исследовали с использованием сканирующего (растрового) электронного микроскопа TESCAN VEGA 3 SBH в Инжиниринговом центре СПбГТИ(ТУ). Анализ полученного ряда микрофотографий свидетельствует о высокой однородности структуры композитов в пределах центральной части каждого образца, поэтому для анализа полученных изображений использовали их центральные фрагменты, соответствующие площади сечения $24 \times 24 \mu \mathrm{m}$ и содержащие достаточно большое для статистической обработки число частиц (300-600). Методика обработки выбранных фрагментов включала следующие операции.

1. Выделение на микрофотографии частиц наполнителя и определение координат их центров масс с помощью программы ImageJ. Для этого во вкладке „Analyze“ в разделе „Set measurements“ был отмечен пункт „центры масс“, после чего в разделе „Analyze particles“ было получено изображение с выделенными и пронумерованными частицами, а также таблицу с координатами $x$ и $y$ каждой частицы.

2. Разбиение анализируемых фрагментов микрофотографий на 64 квадратных ячейки со стороной $3 \mu \mathrm{m}$, с подсчетом количества центров масс, попавших в каждый из таких сегментов. Данная операция была выполнена в табличном редакторе Excel 2016 с использованием функции „И“, проверяющей соответствие координат центров масс координатам сегментов.

3. Статистическая обработка полученных результатов, включающая расчет размаха, среднеквадратичного отклонения и лакунарности $\Lambda$ - параметра, характеризующего неоднородность заполнения пространства изучаемыми объектами $[10,11]$.

$$
\Lambda=\left(\frac{\sigma}{\mu}\right)^{2},
$$

где $\sigma$ и $\mu$ - соответственно стандартное отклонение и среднее значение количества центров масс частиц наполнителя в рассматриваемых сегментах.

Снижение величины данного параметра соответствует уменьшению доли не заполненных частицами наполнителя областей и, соответственно, повышению равномерности заполнения ими пространства.

4. Разбиение анализируемых микрофотографий на концентрические круговые сегменты (слои) относительно центра изображения с шагом изменения радиуса $0.5 \mu \mathrm{m}$, что эквивалентно среднему радиусу частиц. 
Таблица 1. Характеристики распределения центров масс частиц наполнителя по 64 квадратным сегментам

\begin{tabular}{c|c|c|c|c|c|c}
\hline Образец & $\begin{array}{c}\text { Средний размер } \\
\text { частиц, } \mu \mathrm{m}^{2}\end{array}$ & $\begin{array}{c}\text { Общее число } \\
\text { частиц }\end{array}$ & $\begin{array}{c}\text { Число частиц } \\
\text { в ячейке }\end{array}$ & Размах & $\begin{array}{c}\text { Среднее квадратичное } \\
\text { отклонение }\end{array}$ & $\begin{array}{c}\text { Параметр } \\
\text { лакунарности }\end{array}$ \\
\hline Без добавки фуллеренола & 0.3 & 359 & $5.27 \pm 0.86$ & 8 & 2.00 & 0.15 \\
С добавкой фуллеренола & 0.2 & 567 & $8.33 \pm 0.99$ & 10 & 2.38 & 0.08
\end{tabular}

Построение радиальных сегментов выполнялось в табличном редакторе Excel 2016 с помощью функции „ЕСЛИ“, проверяющей соответствие координат заданному условию $x_{i}^{2}+y_{i}^{2}<R_{i}^{2}$, где $x_{i}$ и $y_{i}-$ координаты центров масс частиц, а $R_{i}$ - радиус кругового сегмента (слоя).

5. Определение площади каждого кругового сегмента (слоя) $S\left(\mu \mathrm{m}^{2}\right)$ и количества попавших в него центров масс частиц $N$ с последующим расчетом плотности заполнения слоя по формуле

$$
P=N / S \text {. }
$$

6. Построение радиальных функций распределения $g(r)$, представляющих собой зависимости плотности заполнения сегментов от их радиуса [9], и их анализ с определением следующих характеристик микроструктуры композитов:

- радиуса первой координационной сферы $r_{s}$, соответствующего первому минимуму функции $g(r)$;

- вероятного расстояния между частицами $L$, равного разности между величиной $r_{s}$ и средним радиусом частиц (либо их агломератов) $r: L=r_{s}-r$;

- усредненного координационного числа $Z$ - числа ближайших соседей частицы в координационной сфере с радиусом $r_{s}$. Параметр $Z$ определяли с использованием программы Excel 2016 методом кластеризации посредством подсчета количества ближайших соседей для каждой частицы на расстояниях $r$, определенных из $g(r)$.

7. Определение фрактальной размерности исследуемых композитов (количественной меры плотности заполнения пространства частицами наполнителя) с использованием программы ImageJ по алгоритму box counting $[10,12,13]$. Данный алгоритм основан на методе квадратных сеток - покрытии анализируемого изображения сетками из квадратных ячеек с последовательно уменьшаемым размером стороны $a$ и подсчетом количества ячеек $N(a)$, в которые попал центр масс хотя бы одной из частиц наполнителя. Если получаемая таким образом зависимость $\ln \left(N_{i}\right)=f(a)$ имеет линейный вид, то, согласно степенному соотношению [12],

$$
N(a) \propto \frac{1}{a^{D}},
$$

где $N(a)$ - количество элементов равномерного покрытия (ячеек) с размером $a$. Фрактальная размерность определяется выражением

$$
D=\lim _{a \rightarrow 0} \frac{\ln N(a)}{\ln a}
$$

и может быть рассчитана как модуль тангенса угла наклона соответствующего графика или коэффициента перед $x$ в аппроксимирующем уравнении.

\section{3. Результаты и их обсуждение}

Электронные микрофотографии композитов на основе немодифицированного и модифицированного $\mathrm{BaTiO}_{3}$ и их разбиение на квадратные сегменты размером $3 \times 3 \mu \mathrm{m}$ с выделением центров масс частиц наполнителя приведены на рис. 1, а характеристики распределения частиц наполнителя по квадратным сегментам - в табл. 1.

Анализ полученных данных показывает, что введение фуллеренола в состав наполнителя приводит к росту числа частиц на всем изображении и в среднем по ячейкам примерно в 1.5 раза, что соответствует снижению среднего размера частиц наполнителя в 1.5 раза (с 0.3 до $0.2 \mu \mathrm{m}^{2}$ ). Наиболее значительное изменение наблюдается в отношении лакунарности, которая в результате введения фуллеренола уменьшилась почти в 2 раза (с 0.15 до 0.08). Это свидетельствует о снижении степени агломерации частиц и повышении однородности их распределения в полимере. Вместе с тем, наблюдается незначительное увеличение размаха и стандартного квадратичного отклонения $(\sigma)$ количества частиц в отдельных фрагментах, однако для образца с исходным наполнителем величина $\sigma$ составляет $37.9 \%$ от среднего значения, а в композите с добавкой фуллеренола $-28.3 \%$.

Сопоставление распределения представленных на микрофотографиях частиц по размерам (рис. 2) подтверждает их уменьшение, что характеризуется выраженным смещением максимума распределения. Кроме того, модифицирование наполнителя приводит к изменению характера распределения ячеек по числу частиц, который становится отчетливо полимодальным (рис. 3). Это отражает более сложный характер взаимодействия наполнитель-связующее и наполнитель-наполнитель по сравнению с композитом, содержащим исходный титанат бария. 

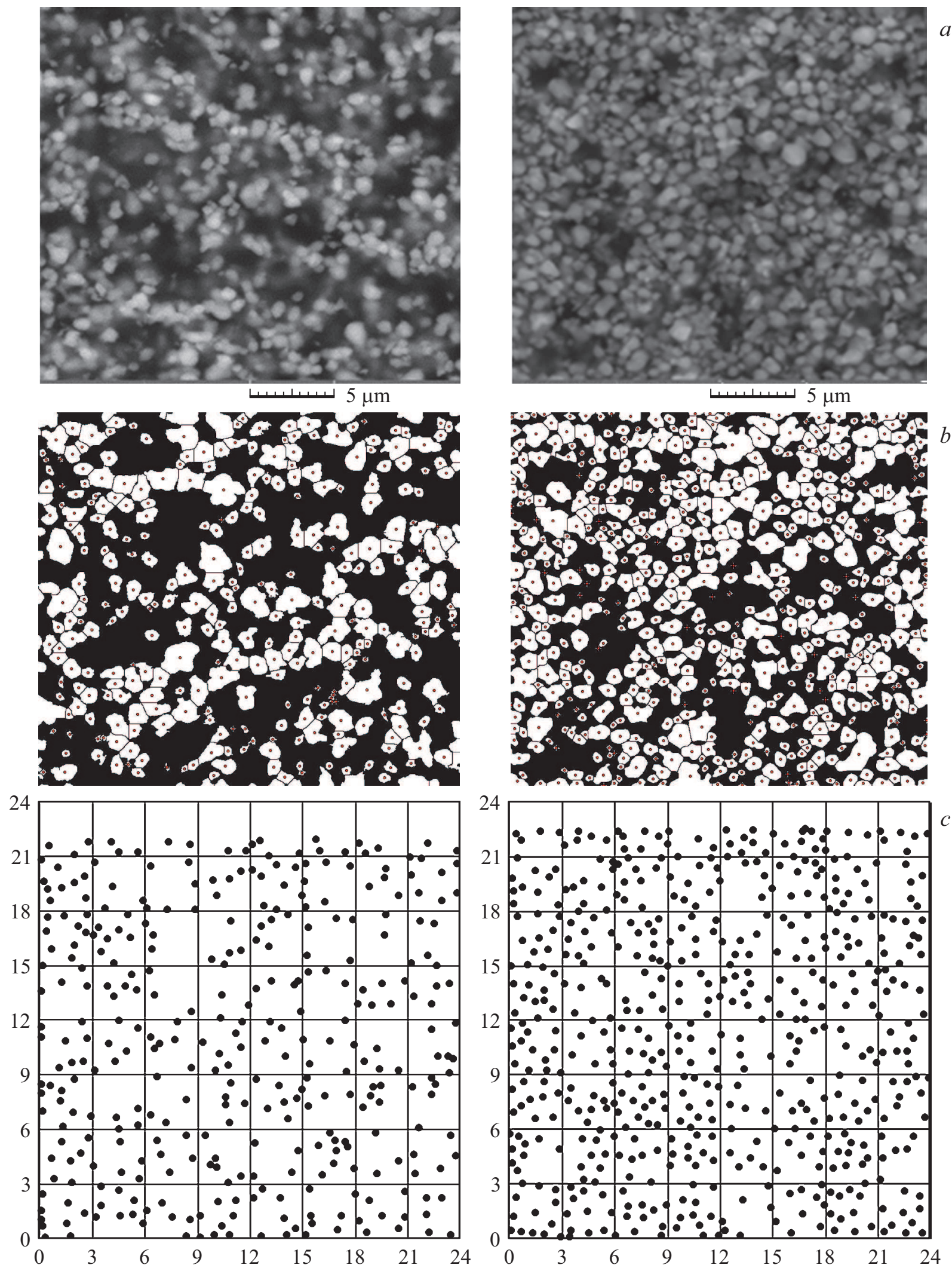

Рис. 1. Электронные микрофотографии $(a)$, их бинаризированное представление с отмеченными центрами масс частиц $(b)$ и распределения центров масс частиц по квадратным ячейкам (c) для композитов с исходным (слева) и модифицированным добавкой $3.2 \mathrm{mg} / \mathrm{g}$ фуллеренола (справа) наполнителем. 


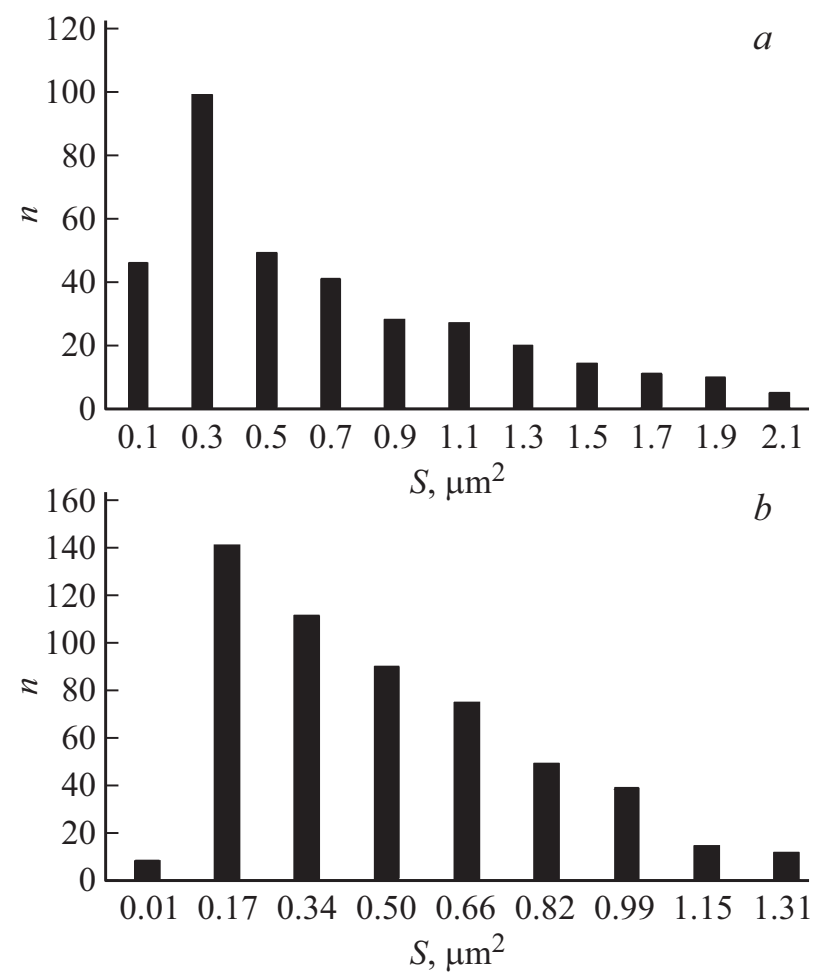

Рис. 2. Распределение числа частиц $(n)$ по размерам (площади сечения $S$ ) в композитах с исходным $(a)$ и модифицированным (b) наполнителем.
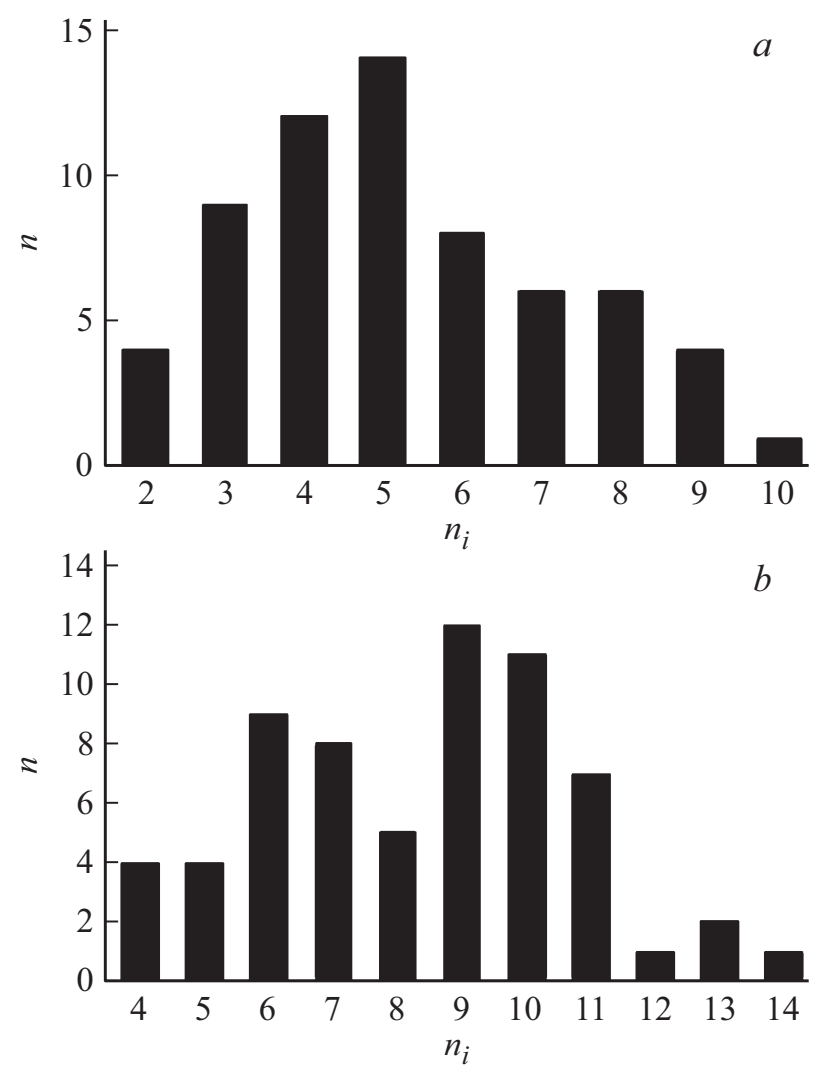

Рис. 3. Распределение числа частиц наполнителя $(n)$ по ячейкам с $i$-м числом частиц $\left(n_{i}\right)$ для композитов с исходным $(a)$ и модифицированным $(b)$ наполнителем.
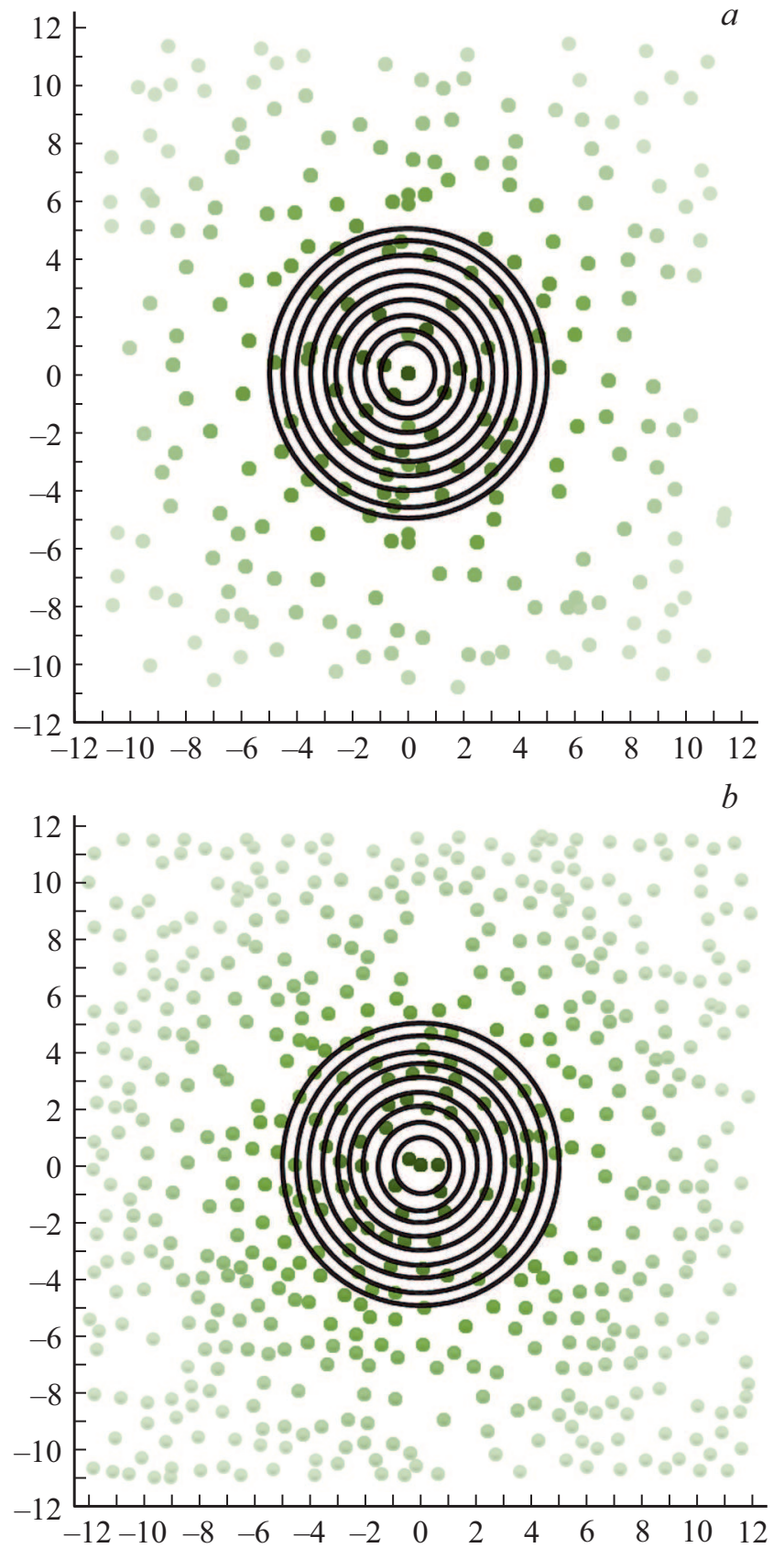

Рис. 4. Распределение частиц по концентрическим круговым сегментам для композитов с исходным $(a)$ и модифицированным $(b)$ наполнителем.

Разбиение анализируемых изображений на концентрические круговые сегменты приведено на рис. 4, а соответствующие радиальные функции распределения - на рис. 5.

На рис. 6 приведены полученные по алгоритму box counting зависимости, используемые для определения фрактальной размерности композитов.

Результаты анализа полученных зависимостей, приведенные в табл. 2, свидетельствуют о том, что модифицирование наполнителя привело к существенному 
Таблица 2. Характеристики микроструктуры композитов, рассчитанные на основе анализа радиальной функции распределения и обработки данных по алгоритму box counting

\begin{tabular}{c|c|c|c|c|c}
\hline Образец & $\begin{array}{c}\text { Радиус первой } \\
\text { координационной } \\
\text { сферы } \mathbf{r}_{s}, \mu \mathrm{m}\end{array}$ & $\begin{array}{c}\text { Наиболее вероятное } \\
\text { расстояние между } \\
\text { частицами } L, \mu \mathrm{m}\end{array}$ & $\begin{array}{c}\text { Среднее } \\
\text { координационное } \\
\text { число } Z\end{array}$ & $\begin{array}{c}\text { Фрактальная } \\
\text { размерность } D\end{array}$ & $\begin{array}{c}\text { Диэлектрическая } \\
\text { проницаемость } \varepsilon\end{array}$ \\
\hline Без добавки фуллеренола & 2.0 & 1.5 & 7 & 1.74 & 50 \\
С добавкой фуллеренола & 1.5 & 1 & 6 & 1.80 & 400
\end{tabular}

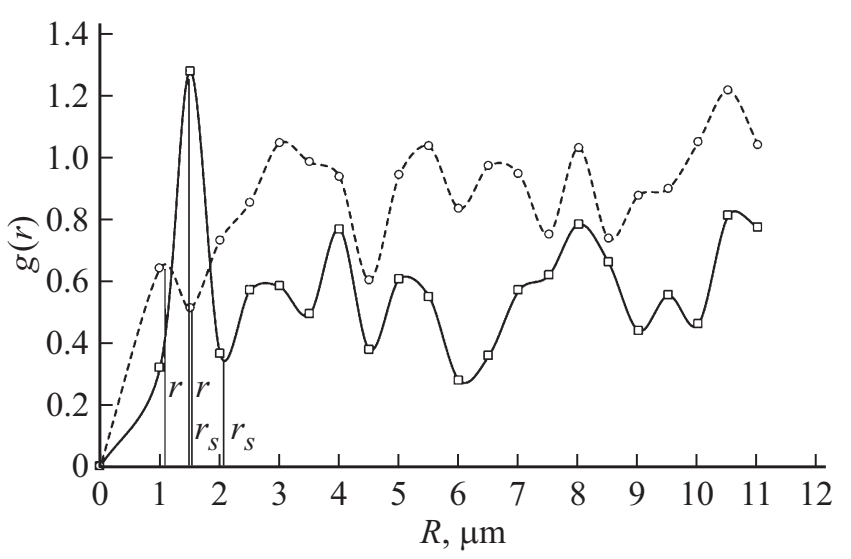

Рис. 5. Радиальные функции распределения частиц наполнителя в композитах с исходным (-) и модифицированным (---) наполнителем.

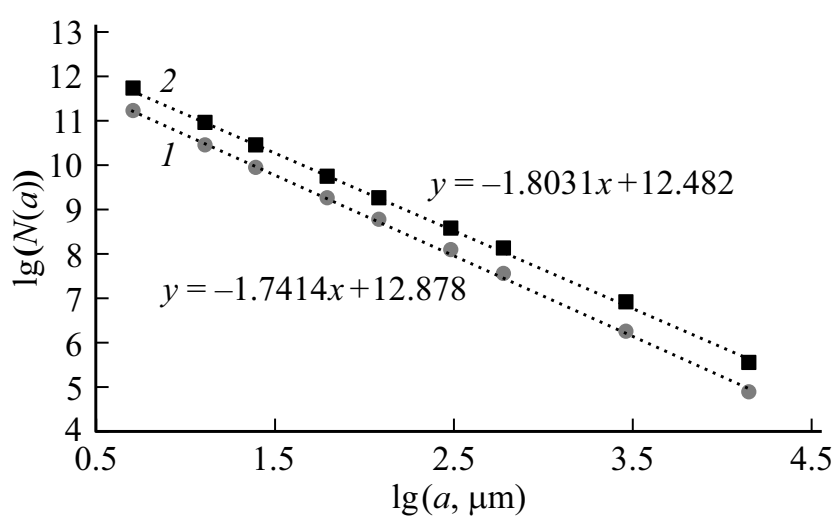

Рис. 6. Логарифмические зависимости числа занятых ячеек $N(a)$ от размера ячейки $(a)$ по данным анализа структуры композитов методом box counting для композитов с исходным (1) и модифицированным (2) наполнителем.

уменьшению радиуса первой координационной сферы и вероятного расстояния между частицами наполнителя в композите, а также уменьшению среднего координационного числа. Такое изменение рассматриваемых характеристик подтверждает снижение степени агломерации частиц и повышение однородности их распределения в матрице. Кроме того, наблюдается некоторое увеличение фрактальной размерности исследуемой системы, что отражает рост плотности заполнения пространства частицами наполнителя и коррелирует с отмеченным выше снижением лакунарности. В целом наблюдаемые изменения характера распределения частиц наполнителя в матрице обусловлены взаимодействием между гидроксильными группами фуллеренола и полимерной матрицы, предотвращающим взаимодействие частиц титаната бария друг с другом и, соответственно, их агломерацию [4]. Сопоставление полученных количественных характеристик микроструктуры исследуемых композитов с их электрическими свойствами позволяет сделать вывод о том, что повышение однородности распределения частиц сегнетоэлектрического наполнителя способствует росту диэлектрической проницаемости исследуемого материала. Достигнутое улучшение диэлектрических свойств делает перспективным применение рассматриваемых материалов в качестве защитных диэлектрических слоев в различных электронных устройствах.

Проведенный анализ позволяет исследовать взаимосвязь „состав-структура-свойства“ и оценить влияние модифицирования поверхности наполнителя на характер его распределения в матрице. Сопоставление результатов рассматриваемых исследований с целевыми свойствами композиционного материала обеспечивает возможность направленного регулирования его целевых характеристик.

\section{4. Заключение}

В результате сравнительного анализа характеристик микроструктуры диэлектрических композитов на основе цианэтилового эфира поливинилового спирта показано, что модифицирование поверхностного слоя используемого в качестве наполнителя титаната бария введением микродобавки фуллеренола приводит к резкому росту диэлектрической проницаемости композитов. Достигнутый эффект обусловлен повышением однородности распределения и снижением степени агломерации частиц сегнетоэлектрического наполнителя в полимерной матрице. В целом на рассмотренном примере продемонстрирован комплексный подход к анализу микроструктуры композитов и других гетерогенных материалов, основанный на определении ряда параметров, характеризующих однородность взаимного расположения и пространственного распределения фаз, включая лакунарность, радиус координационной сферы, усредненное координаци- 
онное число и фрактальную размерность. Сопоставление рассматриваемых параметров со свойствами материалов позволяет прогнозировать их целевые характеристики и оптимизировать условия их изготовления.

\section{Финансирование работы}

Работа выполнена при поддержке гранта РФФИ 20-07-00740.

\section{Конфликт интересов}

Авторы заявляют, что у них нет конфликта интересов.

\section{Список литературы}

[1] М.И. Карякина, В.Е. Попцов. Технология полимерных покрытий. Химия, М. (1983). 336 с.

[2] А.Г. Масловская, Т.К. Барабаш. Вестн. Амурского гос. ун-та 55, 35 (2011).

[3] К.В. Макаренко, Д.А. Илюшкин. Вестн. Брянского гос. техн. ун-та 49, 34 (2016).

[4] М.М. Сычев, Е.С. Васина, С.В. Мякин, Н.Н. Рожкова, Н.Т. Сударь. Конденсированные среды и межфазные границы 16, 354 (2014).

[5] M.M. Sychov, S.V. Mjakin, A.N. Ponyaev, V.V. Belyaev. Adv. Mater. Res. 1117, 147 (2015).

[6] M. Sychov, Y. Nakanishi, E. Vasina, A. Eruzin, S. Mjakin, T. Khamova, O. Shilova, H. Mimura. Chem. Lett. 44, 197 (2015).

[7] С.В. Мякин, В.А. Гарипова, М.М. Сычев. Изв. СПбГТИ (ТУ) 50, 76, 68 (2019).

[8] Д.В. Новиков, Г.К. Ельяшевич, В.К., И.С. Курындин, A. Anzlovar, V. Bukosek. ФTT 56, 390 (2014).

[9] А.Н. Красовский, Д.В. Новиков, Е.С. Васина, П.В. Матвейчикова, М.М. Сычев, Н.Н. Рожкова. ФТТ 57, 2479 (2015).

[10] Д.В. Новиков. ФТТ 60, 1829 (2018).

[11] R.E. Plotnick, R.H. Gardner, R.V. O’Neill. Landscape Ecol 8, 201 (1993).

[12] Д.И. Иудин, Е.В. Копосов. Фракталы: от простого к сложному. ННГАСУ, Н. Новгород (2012). 200 с.

[13] О.В. Чумак. Энтропии и фракталы в анализе данных. НИЦ „Регулярная и хаотическая динамика“, Институт компьютерных исследований, М.-Ижевск (2011). 164 с.

Редактор Т.Н. Василевская 